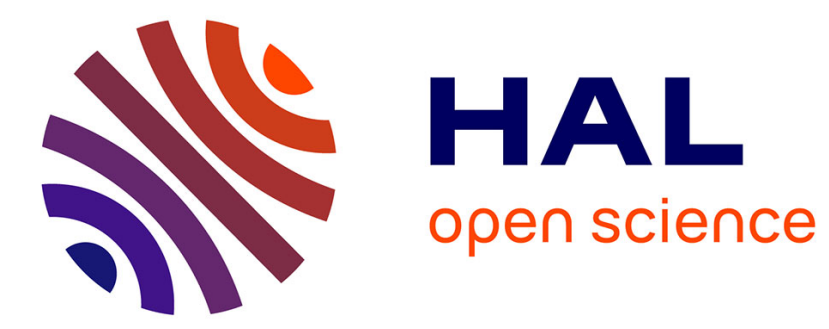

\title{
Internal shear layers from librating objects
}

Stéphane Le Dizès, Michael Le Bars

\section{To cite this version:}

Stéphane Le Dizès, Michael Le Bars. Internal shear layers from librating objects. Journal of Fluid Mechanics, 2017, 826, pp.653-675. hal-01543666

\section{HAL Id: hal-01543666 https://hal-amu.archives-ouvertes.fr/hal-01543666}

Submitted on 23 Jun 2017

HAL is a multi-disciplinary open access archive for the deposit and dissemination of scientific research documents, whether they are published or not. The documents may come from teaching and research institutions in France or abroad, or from public or private research centers.
L'archive ouverte pluridisciplinaire HAL, est destinée au dépôt et à la diffusion de documents scientifiques de niveau recherche, publiés ou non, émanant des établissements d'enseignement et de recherche français ou étrangers, des laboratoires publics ou privés. 


\title{
Internal shear layers from librating objects
}

\author{
By Stéphane LE DIZĖS and Michael LE BARS \\ CNRS, Aix Marseille Univ, Centrale Marseille, IRPHE, Marseille, France
}

(Received 8 June 2017)

In this work, we analyse the internal shear layer structures generated by the libration of an axisymmetric object in an unbounded fluid rotating at a rotation rate $\Omega^{*}$ using direct numerical simulation and small Ekman number asymptotic analysis. We consider weak libration amplitude and libration frequency $\omega^{*}$ within the inertial wave interval $\left(0,2 \Omega^{*}\right)$ such that the fluid dynamics is mainly described by a linear axisymmetric harmonic solution. The internal shear layer structures appear along the characteristic cones of angle $\theta_{c}=\operatorname{acos}\left(\omega^{*} /\left(2 \Omega^{*}\right)\right)$ which are tangent to the librating object at so-called critical latitudes. These layers correspond to thin viscous regions where the singularities of the inviscid solution are smoothed. We assume that the velocity field in these layers is described by the class of similarity solutions introduced by Moore \& Saffman [Phil. Trans. R. Soc. A 264, 597-634 (1969)]. These solutions are characterised by two parameters only: a real parameter $m$, which measures the strength of the underlying singularity, and a complex amplitude coefficient $C_{0}$.

We first analyse the case of a disk for which a general asymptotic solution for small Ekman numbers is known when the disk is in a plane. We demonstrate that the numerical solutions obtained for a free disk and for a disk in a plane are both well-described by the asymptotic solution and by its similarity form within the internal shear layers. For the disk, we obtain a parameter $m=1$ corresponding to a Dirac source at the edge of the disk and a coefficient $C_{0} \propto E^{1 / 6}$ where $E$ is the Ekman number.

The case of a smoothed librating object such as a spheroid is found to be different. By asymptotically matching the boundary layer solution to similarity solutions close to a critical latitude on the surface, we show that the adequate parameter $m$ for the similarity solution is $m=5 / 4$, leading to a coefficient $C_{0} \propto E^{1 / 12}$, that is larger than for the case of a disk for small Ekman numbers. A simple general expression for $C_{0}$ valid for any axisymmetric object is obtained as a function of the local curvature radius at the critical latitude in agreement with this change of scaling. This result is tested and validated against direct numerical simulations.

\section{Introduction}

In a rotating fluid, localized time-harmonic perturbations propagate if their frequency $\omega^{*}$ is smaller than twice the fluid rotation rate $\Omega^{*}$ (Greenspan, 1968). The propagation occurs along conical surfaces of angle $\theta_{c}=\operatorname{acos}\left(\omega^{*} /\left(2 \Omega^{*}\right)\right)$ with respect to the plane normal to the rotation axis. Oscillating singularities travel within the fluid the same way. When these singular surfaces are smoothed by viscosity, they form internal shear layers. The goal of the present work is to analyse the structure and the amplitude of such layers when they are created by the (longitudinal) libration of an axisymmetric object in an open domain.

Longitudinal libration denotes harmonic oscillation of the rotation rate without modification of the rotation axis. It constitutes one of the possible harmonic forcings in 
planetology. Other forcings such as tide, precession, latitudinal libration are also present in most gravitational systems (Le Bars et al., 2015). Longitudinal libration is especially important for so-called synchronized bodies, i.e. bodies locked with their main companion in a given spin-orbit resonance, which is periodically perturbed by gravitational interactions with other bodies. This includes moons and small planets of our solar system, as well as some of the detected exoplanets in extra-solar systems, which orbit close to their star (see e.g. discussion in Cébron et al., 2012). The observed response of those bodies to libration forcing, and more specifically the small changes in the measured spin rate of their surface, are used to remotely investigate their internal composition, showing for instance the presence of a liquid core in Mercury (Margot et al., 2007) and of a global subsurface ocean in Enceladus (Thomas et al., 2016). Libration driven flows may also be of importance in the dynamics and evolution of planetary bodies, notably because of the associated energy dissipation and because of the possibility to generate a magnetic field (see e.g. Le Bars et al., 2015, and references therein). However, the details of harmonic flows excited by libration are still largely unknown and controversial (see e.g. Noir et al., 2009; Koch et al., 2013; Zhang et al., 2013; Klein et al., 2014).

In a close geometry, an external forcing can resonantly excite global modes (Aldridge \& Toomre, 1969). In simple geometries such as the cylinder or the sphere, these modes can be described by so-called inviscid Kelvin modes. Even in these cases, the viscous correction to these modes does exhibit internal shear layers. These layers are associated with corners of the geometry as in a cylinder (McEwan, 1970) or with scaling variations of the viscous boundary layer close to critical latitudes. Kerswell (1995) demonstrated that whether the boundary is concave or convex at the critical latitude, different internal shear layers are expected. From a concave boundary, the boundary layer eruption, which is on a large $\mathrm{O}\left(E^{1 / 5}\right)$ region, is transmitted throughout the interior without modification (Kida, 2011), while it is transmitted and smoothed on a smaller $\mathrm{O}\left(E^{1 / 3}\right)$ width from a convex boundary (Kerswell, 1995). It is this second kind of internal shear layers that will be our interest in the present work. We shall in particular correct the prediction in $\mathrm{O}\left(E^{1 / 6}\right)$ found in the literature for the amplitude of the solution in this layer (e. g. Kerswell, 1995; Calkins et al., 2010).

When the geometry is more complex as in a shell, other types of internal shear layers can be observed. Some of them result from the viscous smoothing of an inviscid attractor (e.g. Rieutord \& Valdettaro, 1997).

Internal shear layers have been first studied in the context of stationary flows. Many works have concerned disks (Stewartson, 1957; Moore \& Saffman, 1969; van de Vooren, 1992) or spheres (Proudman, 1956; Stewartson, 1966; Marcotte et al., 2016). Similarity solutions have been introduced for their description by Moore \& Saffman (1969) for rotating flows, and by Thomas \& Stevenson (1972) for stratified fluids. Their properties have been reviewed by Voisin (2003). Extensions to more general situations can also be found in Stevenson et al. (1974) and Peat (1978). These similarity solutions describe the viscous smoothing in a $\mathrm{O}\left(E^{1 / 3}\right)$ layer of an inviscid singularity propagating along a characteristic line. These solutions depend on a real parameter which characterises the strength of the singularity. This parameter also controls the amplitude of the solution and its decay rate from the source (Machicoane et al., 2015). Surprinsingly, these solutions were also found to be able to describe the fine viscous structure resulting from an attractor (Walton, 1975; Rieutord et al., 2001; Ogilvie, 2005).

The paper is organised as follows. In $\S 2$, the basic equations and the similarity solutions are introduced. The numerical tool is also described. In $\S 3$, we consider the case of a librating disk. We show that the internal shear layers obtained from the numerical simulations are well-described by the similarity solutions derived from the asymptotic 
analysis. In $\S 4$, we analyse the case of librating spheroids. We first perform an asymptotic analysis of the viscous boundary layer in the neighborhood of the critical latitude to compute the characteristics of the similarity solutions. The asymptotic results are then validated using the simulation. In the last section $\S 5$, the consequences of the results are briefly discussed.

\section{Theoretical background}

\subsection{Basic equations}

We consider the flow generated by the libration of an axisymmetric object in a viscous incompressible rotating fluid. The fluid rotates with an angular rotation $\Omega^{*}$ around the symmetry axis $O z$ of the object. The frequency and amplitude of the libration are $\omega^{*}$ and $\varepsilon^{*}$, respectively, such that the angular rotation vector of the object surface can be written as $\Omega_{\mathbf{S}}^{*}(t)=\left(\Omega^{*}+\varepsilon^{*} \cos \left(\omega^{*} t\right)\right) \mathbf{e}_{\mathbf{z}}$. The frequency is chosen in the inertial wave regime $0<\omega^{*}<2 \Omega^{*}$ such that we can define a propagation angle $\theta_{c}$ satisfying $\omega^{*}=2 \Omega^{*} \cos \theta_{c}$. The angle $\theta_{c}$ defines the direction (with respect to the horizontal plane $O x y$ ) of propagation of the inertial wave of frequency $\omega^{*}$. It also corresponds to the inclination angle of the internal shear layers.

We shall be interested in the internal shear layers that are generated from disks and spheroids. These internal shear layers are issued from the border of the disk, or from the boundary points of the spheroid corresponding to a critical latitude, that is such that the direction normal to the object makes an angle $\theta_{c}$ with respect to the rotation axis $O z$.

In the following, time and space variables are non-dimensionalized using $1 / \Omega^{*}$ and the distance $r_{c}^{*}$ of the internal shear layer source to the rotation axis, respectively. In non-dimensionalized form, the libration frequency and amplitude are then written as

$$
\omega=2 \cos \theta_{c}, \varepsilon=\varepsilon^{*} / \Omega^{*} .
$$

The dynamic, which is assumed to remain axisymmetrical, is analysed in a cylindrical rotating frame such that the internal shear layer source is located on a ring at $\mathbf{x}_{\mathbf{c}}=$ $\left(r_{c}, z_{c}\right)=(1,0)$. This choice implicitly assumes that the source is not on the rotation axis.

Our objective is to describe the internal shear layer structures of the harmonic response to libration in the limit of small viscosity. Both the libration amplitude $\varepsilon$ and the Ekman number

$$
E=\frac{\nu}{\Omega^{*}\left(r_{c}^{*}\right)^{2}},
$$

where $\nu$ is the kinematic viscosity of the fluid are therefore assumed to be small.

If we write the harmonic response of the velocity and pressure field as

$$
\left(\mathbf{v}_{t}, P_{t}\right)=\Re e\left\{\varepsilon(\mathbf{v}, p) e^{-\mathrm{i} \omega t}\right\},
$$

its amplitudes $\mathbf{v}$ and $p$ satisfy in the rotating cylindrical frame the equations:

$$
\begin{aligned}
-2 \mathrm{i} \cos \theta_{c} \mathbf{v}+2 \mathbf{e}_{\mathbf{z}} \wedge \mathbf{v} & =-\nabla p+E \nabla^{2} \mathbf{v}, \\
\nabla \cdot \mathbf{v} & =0 .
\end{aligned}
$$

The boundary conditions at infinity and on the object impose

$$
\begin{aligned}
& \mathbf{v}(\mathbf{x})=r \mathbf{e}_{\phi}, \text { on the object surface, } \\
& \mathbf{v}(\mathbf{x}) \rightarrow 0 \text { as }|\mathbf{x}| \rightarrow \infty
\end{aligned}
$$




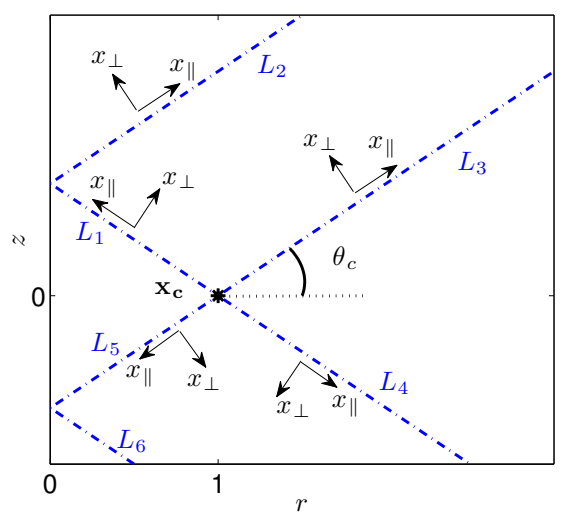

Figure 1. Definitions of the lines $L_{j}, j=1,2,3,4,5,6$ and associated coordinate systems in the $(r, z)$ plane.

Both $\mathbf{v}$ and $p$ are assumed to depend on $r$ and $z$ only.

\subsection{Similarity solution for the description of internal shear layers}

As already mentioned above, similarity solutions were introduced by Moore \& Saffman (1969) and Thomas \& Stevenson (1972). Here, we review some of their properties and introduce our notations. Additional information can be found in Voisin (2003).

By definition, the source point of the similarity solution is $\mathbf{x}_{\mathbf{c}}=(1,0)$. For a given frequency $\omega$, the propagation occurs along the characteristic lines $L_{j}, j=1,2,3,4,5$, as indicated in figure 1 . These lines, which actually correspond to sections of cones, make an angle $\theta_{c}$, defined by (2.1), with respect to the horizontal plane. The four lines $L_{1}$, $L_{3}, L_{4}$ and $L_{5}$ are the four possible directions of emission from $\mathbf{x}_{\mathbf{c}}$. The line $L_{2}$ (and similarly $L_{6}$ ) is different: it is the continuation of the line $L_{1}$ after reflection on the axis of symmetry. Finding the properties of the similarity solution along this line is then slightly more complicated: it requires an analysis of the reflection process on the axis.

We therefore start by the other lines and focus on one of them: $L_{1}$. Along $L_{1}$, we define a local frame $\left(\mathbf{e}_{\|}, \mathbf{e}_{\perp}\right)$ and a local coordinate system $\left(x_{\|}, x_{\perp}\right)$ such that $r \mathbf{e}_{r}+z \mathbf{e}_{z}=$ $\mathbf{e}_{r}+x_{\|} \mathbf{e}_{\|}+x_{\perp} \mathbf{e}_{\perp}$ with

$$
\begin{array}{r}
\mathbf{e}_{\|}=-\mathbf{e}_{r} \cos \theta_{c}+\mathbf{e}_{z} \sin \theta_{c}, \\
\mathbf{e}_{\perp}=\mathbf{e}_{r} \sin \theta_{c}+\mathbf{e}_{z} \cos \theta_{c} .
\end{array}
$$

In this frame, equations $(2.4 \mathrm{a}, \mathrm{b})$ can be written as

$$
\begin{aligned}
\left(-2 \mathrm{i} \cos \theta_{c}-E \Delta\right) v_{\|} & =-2 \cos \theta_{c} v_{\phi}-\frac{\partial p}{\partial x_{\|}}, \\
\left(-2 \mathrm{i} \cos \theta_{c}-E \Delta\right) v_{\perp} & =2 \sin \theta_{c} v_{\phi}-\frac{\partial p}{\partial x_{\perp}}, \\
\left(-2 \mathrm{i} \cos \theta_{c}-E \Delta\right) v_{\phi} & =-2 \sin \theta_{c} v_{\perp}+2 \cos \theta_{c} v_{\|}, \\
\frac{\partial v_{\perp}}{\partial x_{\perp}}+\frac{\partial v_{\|}}{\partial x_{\|}} & =2 \cos \theta_{c} \frac{v_{\|}}{r}-2 \sin \theta_{c} \frac{v_{\perp}}{r} .
\end{aligned}
$$

Introducing the local transverse scale $\eta_{\perp}=x_{\perp} / E^{1 / 3}$ and the ansatz

$$
v_{\|}=\frac{u_{\|}^{(0)}}{\sqrt{r}}, \quad v_{\perp}=E^{1 / 3} \frac{u_{\perp}^{(1)}}{\sqrt{r}}, \quad v_{\phi}=\frac{v_{\phi}^{(0)}}{\sqrt{r}}, \quad p=E^{1 / 3} \frac{p^{(1)}}{\sqrt{r}}
$$


we obtain the leading order equations

$$
\begin{array}{r}
v_{\phi}^{(0)}=\mathrm{i} v_{\|}^{(0)}, \\
\frac{\partial p^{(1)}}{\partial \eta_{\perp}}=2 \mathrm{i} \sin \theta_{c} v_{\|}^{(0)}, \\
\frac{\partial v_{\perp}^{(1)}}{\partial \eta_{\perp}}=-\frac{\partial v_{\|}^{(0)}}{\partial x_{\perp}}-\cos \theta_{c} \frac{v_{\|}^{(0)}}{2 r}, \\
i \frac{\partial^{3} v_{\|}^{(0)}}{\partial \eta_{\perp}^{3}}+2 \sin \theta_{c} \frac{\partial v_{\|}^{(0)}}{\partial x_{\|}}=0 .
\end{array}
$$

Similarity solutions are obtained by searching $v_{\|}^{(0)}$ in the form

$$
v_{\|}^{(0)}=C_{0} H_{m}\left(x_{\|}, \zeta\right)=C_{0}\left(\frac{x_{\|}}{2 \sin \theta_{c}}\right)^{-m / 3} h_{m}(\zeta),
$$

where $m$ is a real number, $C_{0}$ a complex constant and

$$
\zeta=\eta_{\perp}\left(\frac{2 \sin \theta_{c}}{x_{\|}}\right)^{1 / 3} .
$$

The function $h_{m}$ is found to satisfy

$$
3 h_{m}^{\prime \prime \prime}+\mathrm{i} \zeta h_{m}^{\prime}+\mathrm{i} m h_{m}=0 .
$$

As shown by Moore \& Saffman (1969), the solution which is bounded for $m>0$ is a multiple of

$$
h_{m}(\zeta)=\frac{e^{-\mathrm{i} m \pi / 2}}{(m-1) !} \int_{0}^{+\infty} e^{\mathrm{i} p \zeta-p^{3}} p^{m-1} d p
$$

The normalization of $h_{m}$ has been chosen such that

$$
\begin{aligned}
& H_{m}\left(x_{\|}, \zeta\right) \sim \eta_{\perp}^{-m}, \\
& H_{m}\left(x_{\|}, \zeta\right) \underset{\zeta \rightarrow+\infty}{\sim} \underset{\zeta \rightarrow-\infty}{\sim}\left(-\eta_{\perp}\right)^{-m} e^{-\mathrm{i} m \pi} .
\end{aligned}
$$

The other components can be deduced from (2.9a-c). Here we shall only use the expression for the azimuthal velocity:

$$
v_{\phi}^{(0)}=\mathrm{i} C_{0} H_{m}\left(x_{\|}, \zeta\right)
$$

The above analysis applies to $L_{1}$. A similar analysis can be performed on the other lines $L_{3}, L_{4}$ and $L_{5}$. With the definition of the coordinate system shown in figure 1 , we obtain the same expression (2.10) for $v_{\|}^{(0)}$ with $\zeta$ defined by (2.11) for all these lines. However, (2.15) is valid on the lines $L_{1}$ and $L_{5}$ only. On the symmetric lines $L_{3}$ and $L_{4}$, we must use

$$
v_{\phi}^{(0)}=-\mathrm{i} C_{0} H_{m}\left(x_{\|}, \zeta\right) .
$$

As shown in appendix, on $L_{2}$, we should use the formula

$$
v_{\|}^{(0)}=\mathrm{i} C_{0} H_{m}\left(x_{\|}, \zeta\right), \quad v_{\phi}^{(0)}=C_{0} H_{m}\left(x_{\|}, \zeta\right),
$$

with $x_{\|}=(r+1) \cos \theta_{c}+z \sin \theta_{c}$ (that is counted from a symmetric source at $(-1,0)$ ). 


\subsection{Description of the numerical method}

Axisymmetric direct numerical simulations are performed with the finite-elements commercial software Comsol Multiphysics. Incompressible Navier-Stokes equations are solved for a Newtonian fluid in the frame of reference rotating at the mean angular velocity $\Omega^{*}$. The length scale in the numerics is the axis distance to the point where the characteristic line $L_{4}$ crosses the equator. Considering the axial and equatorial symmetries of the problem, the fluid domain then corresponds to the upper-right quarter of a sphere of radius 4 (see figure 2). Boundary conditions are stress-free at the spherical radial coordinate $\rho=4$, no-slip with imposed libration azimutal velocity $\mathbf{v}=\varepsilon r \cos (\omega t) \mathbf{e}_{\phi}$ at the object boundary, and symmetry conditions along the rotation axis. Regarding the librating disk problem, we consider 2 configurations for the boundary conditions along the equatorial plane: either $\mathbf{v}=\mathbf{0}$, which we refer to as the "disk in the plane" configuration, or symmetry conditions (that is $v_{z}=\partial_{z} v_{r}=\partial_{z} v_{\phi}=0$ at $z=0$ outside the object), which we refer to as the "free disk" configuration. Symmetry conditions are also used for all the spheroidal geometries. From $\rho=2$ to $\rho=4$, the viscosity increases exponentially with the radius squared by a factor about 2000 , so as to damp outgoing propagating waves: any wave reflected from the outer boundary is then negligible. The mesh is made of triangular standard Lagrange elements of type P3-P2 (i.e. cubic for the velocity field and quadratic for the pressure field). It is strongly refined close to the boundaries and along the characteristic lines, where rapid variations of the velocity field are expected (see figure 2). The total number of degrees of freedom ranges between 800000 and 1.2 million, depending on the geometry of the librating object. At each time step, the system is solved with the sparse direct linear solver PARDISO and the backward difference formulae (BDF) temporal solver with a maximal order 2 . The time step is limited to $1 / 150$ of the libration period. No stabilization technique is used. Starting from $\mathbf{v}=\mathbf{0}$ everywhere in the fluid at time $t=t_{i}=-\pi /(2 \omega)$, the computation is first run during 250 libration cycles so as to reach a steady state; the 5 next oscillations are then recorded and analyzed. We have checked that our results are not significantly affected by changing the grid, the size of the domain, the coefficient of the viscous exponential increase, or the maximum time step.

\section{Libration of a disk}

\subsection{Summary of the asymptotic results for the disk in a plane}

Le Dizès (2015) provided an asymptotic expression of the solution for the librating disk in a plane in the limit of small Ekman numbers. In the inertial wave regime $(0<\omega<2)$, he found, using results by Tilgner (2000) that, outside the boundary layer on the disk $(z \gg \sqrt{E})$, the velocity amplitude of the harmonic solution can be written with the present notation as

$$
\mathbf{u}=A\left(2 \mathbf{u}_{D}-\mathbf{u}_{R}\right),
$$

with

$$
A=\frac{\sqrt{E}}{2 \sin \theta_{c}} e^{\mathrm{i} \frac{\pi}{4}-\mathrm{i} \frac{\theta_{c}}{2}},
$$

where the two contributions $\mathbf{u}_{D}$ and $\mathbf{u}_{R}$ correspond to the solutions generated from a uniform axial oscillating forcing on the disk of unitary amplitude and from a Dirac oscillating ring source of axial flow on the disk edge, respectively. These contibutions are 


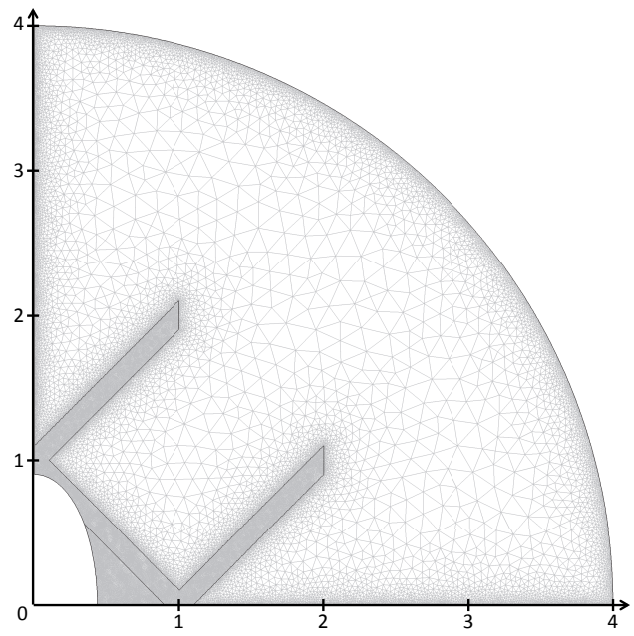

Figure 2. Geometry and grid of the numerical simulation, shown here for a prolate spheroid of eccentricity $\sqrt{2 / 3}$.

given by

$$
\mathbf{u}_{D}=\left\{\begin{array}{l}
u_{D}=-\mathrm{i} \frac{\cos \theta_{c}}{\sin \theta_{c}} I_{D}^{(1)} \\
v_{D}=-\frac{1}{\sin \theta_{c}} I_{D}^{(1)} \\
w_{D}=I_{D}^{(0)}
\end{array} \quad \text { and } \quad \mathbf{u}_{R}=\left\{\begin{array}{l}
u_{R}=-\mathrm{i} \frac{\cos \theta_{c}}{\sin \theta_{c}} I_{R}^{(1)} \\
v_{R}=-\frac{1}{\sin \theta_{c}} I_{R}^{(1)} \\
w_{R}=I_{R}^{(0)}
\end{array}\right.\right.
$$

with

$$
\begin{aligned}
I_{D}^{(m)} & =\int_{0}^{\infty} J_{1}(k) J_{m}(k r) e^{\mathrm{i} k \tilde{z}} e^{-\tilde{E} k^{3} \tilde{z}} d k, \\
I_{R}^{(m)} & =\int_{0}^{\infty} k J_{0}(k) J_{m}(k r) e^{\mathrm{i} k \tilde{z}} e^{-\tilde{E} k^{3} \tilde{z}} d k,
\end{aligned}
$$

where

$$
\tilde{z}=\frac{\cos \theta_{c}}{\sin \theta_{c}} z, \quad \tilde{E}=\frac{E}{2 \cos \theta_{c} \sin ^{4} \theta_{c}} .
$$

Without the diffusing factor $e^{-\tilde{E} k^{3} \tilde{z}}$ the integrals $I_{D}^{(m)}$ and $I_{R}^{(m)}$ are singular on the characteristic lines $L_{j}$ shown in figure 1 . These lines correspond to the conical structure along which the singularity at the edge of the disk propagates. Close to these lines, the functions $I_{D}^{(m)}$ and $I_{R}^{(m)}$ take a particular form as $E$ goes to zero. The function $I_{D}^{(m)}$ is $O(-\log (E))$ while $I_{R}^{(m)}$ becomes $O\left(E^{-1 / 3}\right)$. The part associated with the ring source therefore provides the dominant contribution to the solution. Close to each line, the velocity field is found to be mainly aligned along with the conical structure. The two velocity components $v_{\|}$and $v_{\phi}$ (aligned along with $L_{j}$ and the azimut, respectively) are found to be identical albeit a phase factor. Around the line $L_{1}$, Le Dizès (2015) obtained 


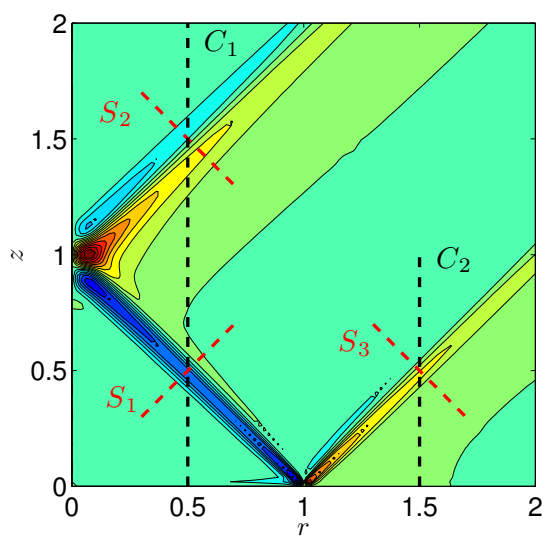

Figure 3. Contour of the azimuthal velocity $v_{\phi}$ in the $(r, \tilde{z})$ plane of the librating disk in the plane obtained from the numerical simulation at $t=501.5 \pi / \omega$ for $E=10^{-5}, \varepsilon=10^{-4}, \omega=\sqrt{2}$. The sections $C_{k}, k=1,2$ and $S_{j}, j=1,2,3$ where the profiles are analysed in figures 4 and 5 are also indicated.

that the solution can be written as (2.8) with

$$
v_{\|}^{(0)}=-\frac{\sqrt{E}}{4 \sin \theta_{c}} e^{\mathrm{i} \frac{\pi}{4}-\mathrm{i} \frac{\theta_{c}}{2}}\left(\frac{2 \sin ^{2} \theta_{c}}{3 E z}\right)^{1 / 3} \mathbf{H i}\left(\mathrm{i} x_{\perp}\left(\frac{2 \sin ^{2} \theta_{c}}{3 E z}\right)^{1 / 3}\right) .
$$

If we recall that the Scorer function $\mathbf{H i}$ can be expressed in term of $h_{1}$ as

$$
\mathbf{H i}(s)=\frac{3^{1 / 3} \mathrm{i}}{\pi} h_{1}\left(-\mathrm{i} 3^{1 / 3} s\right)
$$

and that $z \sim \sin \theta_{c} x_{\|}$close to $L_{1}$, expression (3.6) is found to be of the form (2.10) with

$$
\begin{aligned}
m & =1, \\
C_{0} & =\frac{E^{1 / 6}}{4 \pi \sin \theta_{c}} e^{-\mathrm{i} \frac{\pi}{4}-\mathrm{i} \frac{\theta_{c}}{2}} .
\end{aligned}
$$

The same expressions are obtained for the other lines $L_{j}, j=2,3,4,5,6$.

\subsection{Numerical results}

In this section, we provide numerical results for both the disk in a plane and the disk in a free space. The numerical solution is analysed after 250 periods such that transience has disappeared and a periodic regime has been reached. For the value $\varepsilon=10^{-4}$ that we consider, the solution is dominated by the harmonic response. A small Ekman number $E=10^{-5}$ is also chosen such that the solutions can be compared to the asymptotic expressions given in the previous section. We have chosen a fixed normalized frequency $\omega=\sqrt{2}$ such that $\theta_{c}=\pi / 4$.

In figure 3, a typical contour plot of the azimuthal velocity is shown in the $(r, z)$ plane at a given time for the case of the free disk. In figure 4, the velocity components of the numerical solutions obtained for a free disk and for a disk in a plane are plotted as a function of $z$ in the vertical sections $C_{1}$ and $C_{2}$ corresponding to $r=1 / 2$ and $r=3 / 2$ respectively. The asymptotic solution (3.1) is also plotted in these graphs at the same instant. We can see that the free disk and the disk in the plane solutions are very close to each other and well described by the asymptotic solution. 

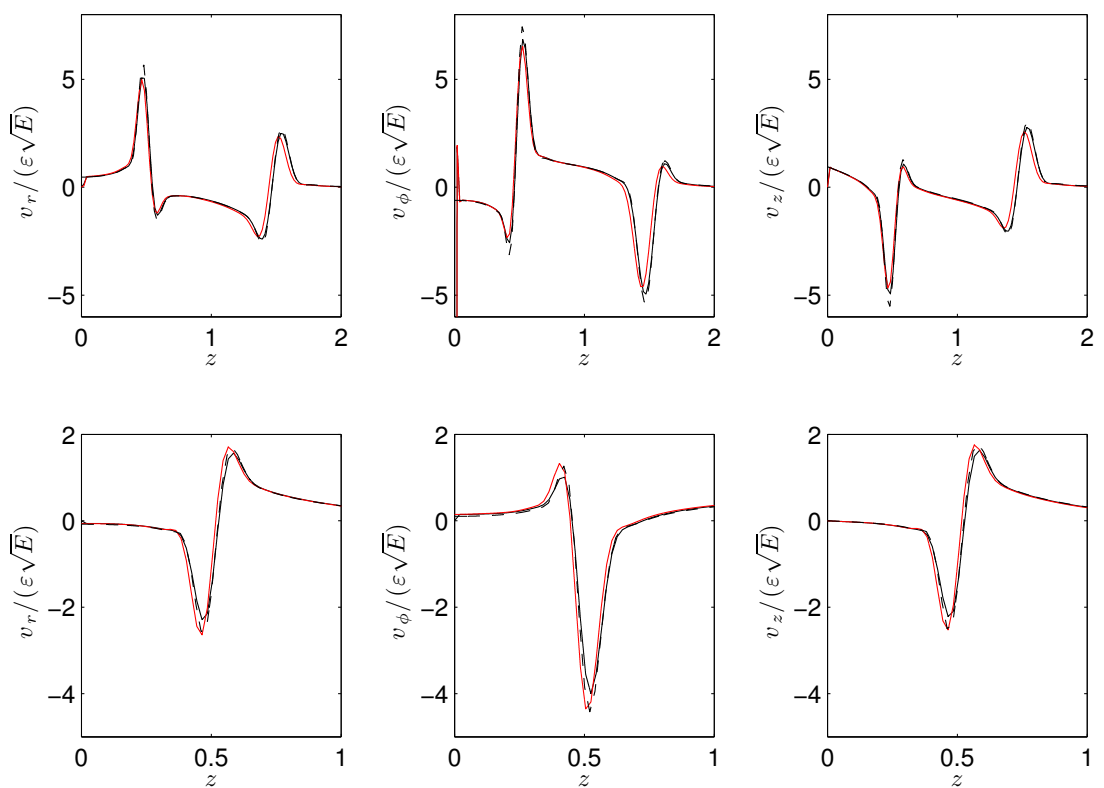

FiguRE 4 . Velocity profiles (left: $v_{r}$, centre: $v_{\phi}$, right: $v_{z}$ ) normalized by $\varepsilon \sqrt{E}$ along the vertical cut at $r=0.5$ (top) and at $r=1.5$ (bottom) for $E=10^{-5}, \varepsilon=10^{-4}, \omega=\sqrt{2}$ at $t_{o}=508.9 \pi / \omega$. Solid lines are numerical results (in black the disk in the plane, in red the free disk) while dashed lines are asymptotic results for the disk in the plane.
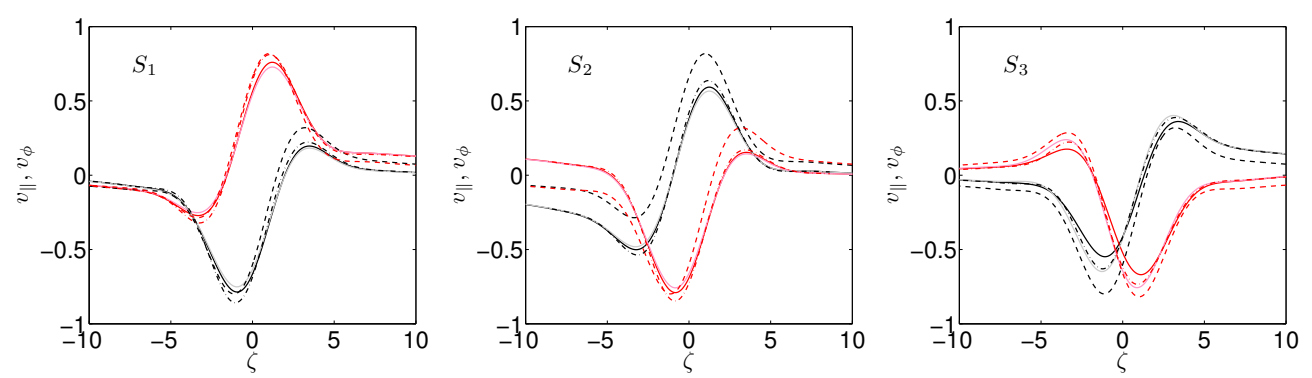

Figure 5. Velocity profiles across the internal shear layer in the sections $S_{1}$ (left), $S_{2}$ (centre) and $S_{3}$ (right) (see figure 3) at time $t_{o}=508.9 \pi / \omega$ for $E=10^{-5}, \varepsilon=10^{-4}, \omega=\sqrt{2}$. Red $\left(v_{\phi}\right)$ and black $\left(v_{\|}\right)$curves are for the disk in the plane, pink $\left(v_{\phi}\right)$ and grey $\left(v_{\|}\right)$are for the free disk. Solid lines are numerical results. The other lines are asymptotic results. Dash-dotted lines: asymptotic formula (3.1) with ring and disk contributions. Dashed lines: similarity solution constructed using $(3.8 \mathrm{a}, \mathrm{b})$. All the solutions have been renormalized by the factor $A=\varepsilon\left|C_{0}\right|\left(2 \sin \theta_{c} / x_{\|}\right)^{1 / 3} / \sqrt{r}$ with $C_{0}$ given by $(3.8 \mathrm{~b})$.

The similarity structure of the numerical solutions is also analysed in figure 5 . In this figure, we plot $v_{\phi}$ and $v_{\|}$, normalized by the amplitude factor $A=\varepsilon\left|C_{0}\right|\left(2 \sin \theta_{c} / x_{\|}\right)^{1 / 3} / \sqrt{r}$ with respect to the similarity variable $\zeta$ defined in (2.11) with the expression (3.8b) for $C_{0}$. We consider the numerical solutions in the sections $S_{1}, S_{2}$ and $S_{3}$ indicated in figure 3 at the same time. These solutions are compared to the theorerical predictions, which 

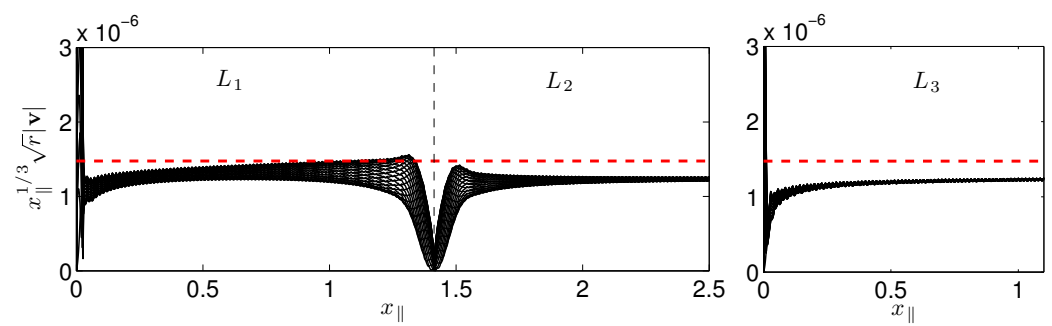

FiguRE 6 . Norm of the velocity vector along the characteristic lines $L_{j}$ normalized by $r^{-1 / 2} x_{\|}^{-1 / 3}$ for a libration disk in the plane. $E=10^{-5}, \varepsilon=10^{-4}, \omega=\sqrt{2}, \theta_{c}=\pi / 4$. The solid black lines are the numerical results obtained for time $\omega t /(2 \pi)=250: 1 / 25: 251$. The dashed line is expression (3.11).

can be written after the renormalisation as

$$
V_{\|}^{S S}=\Re e\left(h_{1}(\zeta) e^{-\mathrm{i} \varphi_{\|}}\right), \quad V_{\phi}^{S S}=\Im m\left(h_{1}(\zeta) e^{-\mathrm{i} \varphi_{\phi}}\right),
$$

where

$$
\begin{aligned}
& \varphi_{\|}=\varphi_{\phi}=\omega t_{o}+3 \pi / 8 \text { for } S_{1}, \\
& \varphi_{\|}=\varphi_{\phi}+\pi=\omega t_{o}-\pi / 8 \text { for } S_{2}, \\
& \varphi_{\|}=\varphi_{\phi}+\pi=\omega t_{o}+3 \pi / 8 \text { for } S_{3} .
\end{aligned}
$$

In figure 6 , the solutions are analysed along the lines $L_{j}$. We plot the norm of the velocity vector, normalized by $1 /\left(x_{\|}^{1 / 3} \sqrt{r}\right.$ ), along the lines $L_{j}$ (that is for $x_{\perp}=0$ ) as a function of $x_{\|}$for different times. The theory predicts that this factor should be constant for all times and equal to

$$
\left.|\mathbf{v}| x_{\|}^{1 / 3} \sqrt{r} \sim \varepsilon\left|h_{1}(0)\right|\left|C_{0}\right|\left(2 \sin \theta_{c}\right)\right)^{1 / 3} \approx 0.0895 \frac{\varepsilon E^{1 / 6}}{\left(\sin \theta_{c}\right)^{2 / 3}} .
$$

This prediction has been indicated as a red dashed line in figure 6 . We do observe that the numerical results follow relatively well the theoretical prediction. The departure between $L_{1}$ and $L_{2}$ corresponds to the region close to the rotation axis where the theory is known to be not applicable. We observe that the numerics provide systematically smaller values than the theory, especially on the line $L_{3}$. In the future, it would be interesting to consider smaller Ekman numbers in the numerics to check that this discrepancy is indeed associated with the not sufficiently small value of the Ekman number.

\section{Libration of a spheroid}

In this section, we analyze the flow generated by the libration of a spheroid. We have no asymptotic solution for this geometry but we suspect that the flow structure around the characteristic rays tangent to the spheroid corresponds to one of the similarity solutions described in $\S 2.2$. To obtain the parameter $m$ and the amplitude factor $C_{0}$ of the solution, we perform an asymptotic analysis close to the critical latitude $\mathbf{x}_{\mathbf{c}}$. More precisely, these parameters are obtained by the condition of matching close to $\mathbf{x}_{\mathbf{c}}$ of the boundary layer solution on the object with the similarity solution. The theoretical result is then tested and validated using direct numerical simulations. 


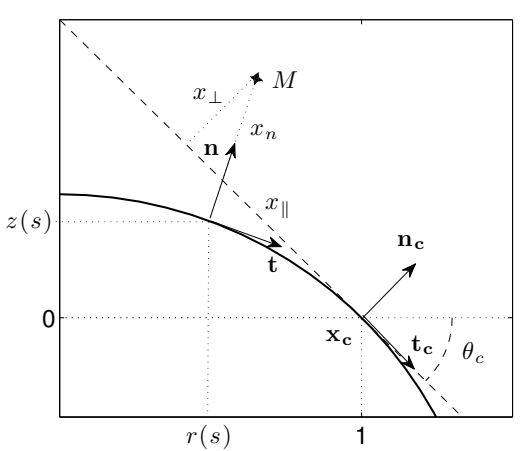

(a)

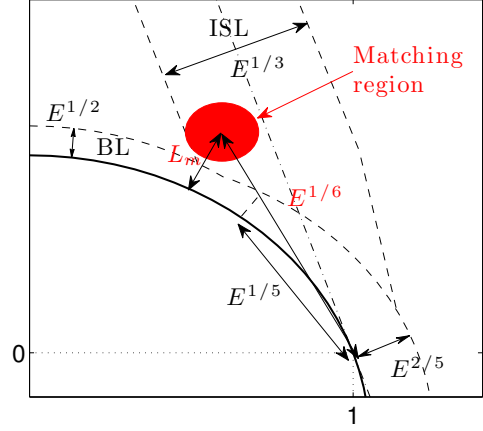

(b)

FiguRE 7. (a) Definition of the coordinate systems near $\mathbf{x}_{\mathbf{c}}$. (b) Sketch of the different regions of the asymptotic analysis. The region of matching is at $O\left(E^{1 / 6}\right)$ from $\mathbf{x}_{\mathbf{c}}$ within the internal shear layer (ISL) but outside the boundary layer (BL), i.e. at a distance $L_{m}$ from the boundary satisfying $E^{5 / 12} \ll L_{m} \ll E^{1 / 3}$.

\subsection{Asymptotic analysis close to $\mathbf{x}_{\mathbf{c}}$}

\subsubsection{Boundary layer solution}

In this section, we consider an arbitrary axisymmetric convex object librating and rotating around its axis of symmetry $O z$. We analyse the solution in a plane $\Sigma$ containing this axis $O z$. In this plane, we assume that the object surface is described by a smooth curve $(r(s), z(s))$, with tangential and normal vectors defined by

$$
\mathbf{t}=\frac{r^{\prime} \mathbf{e}_{\mathbf{r}}+z^{\prime} \mathbf{e}_{\mathbf{z}}}{\alpha}, \quad \mathbf{n}=\frac{-z^{\prime} \mathbf{e}_{\mathbf{r}}+r^{\prime} \mathbf{e}_{\mathbf{z}}}{\alpha}
$$

with

$$
\alpha=\sqrt{r^{\prime 2}+z^{\prime 2}}
$$

where the prime denotes derivative with respect to $s$. The surface being convex, any point $M(\xi, \chi)$ in the fluid (outside the object) is identified by a curvilinear abscissa $s$ and a distance $x_{n}$ to the surface such that $O M=\xi \mathbf{e}_{\mathbf{r}}+\chi \mathbf{e}_{\mathbf{z}}=r(s) \mathbf{e}_{\mathbf{r}}+z(s) \mathbf{e}_{\mathbf{z}}+x_{n} \mathbf{n}$ [see figure $7(\mathrm{a})]$. We assume that the critical latitude $\mathbf{x}_{\mathbf{c}}$ on the surface is reached at $s=s_{c}$. At such a point, the tangential and normal vectors are given by

$$
\mathbf{t}_{c}=\cos \theta_{c} \mathbf{e}_{\mathbf{r}}-\sin \theta_{c} \mathbf{e}_{\mathbf{z}}, \quad \mathbf{n}_{c}=\sin \theta_{c} \mathbf{e}_{\mathbf{r}}+\cos \theta_{c} \mathbf{e}_{\mathbf{z}} .
$$

We therefore have the following equalities:

$$
\begin{gathered}
r\left(s_{c}\right)=r_{c}=1, \quad z\left(s_{c}\right)=z_{c}=0, \\
r^{\prime}\left(s_{c}\right)=r_{c}^{\prime}=\alpha_{c} \cos \theta_{c}, \quad z^{\prime}\left(s_{c}\right)=z_{c}^{\prime}=-\alpha_{c} \sin \theta_{c} .
\end{gathered}
$$

Introducing the boundary layer variable $\eta=x_{n} / \sqrt{E}$ and the following ansatz for the pressure and velocity components along $\mathbf{t}, \mathbf{n}$ and $\mathbf{e}_{\phi}$ :

$$
p=\sqrt{E} \bar{p}^{(1)}(s, \eta), v_{t}=\bar{v}_{t}^{(0)}(s, \eta), v_{n}=\sqrt{E} \bar{v}_{n}^{(1)}(s, \eta), v_{\phi}=\bar{v}_{\phi}^{(0)}(s, \eta),
$$

we get from $(2.4 \mathrm{a}, \mathrm{b})$

$$
\left(-2 \mathrm{i} \cos \theta_{c}-\frac{\partial^{2}}{\partial \eta^{2}}\right) \bar{v}_{t}^{(0)}-2 \frac{r^{\prime}}{\alpha} \bar{v}_{\phi}^{(0)}=0
$$




$$
\begin{gathered}
\left(-2 \mathrm{i} \cos \theta_{c}-\frac{\partial^{2}}{\partial \eta^{2}}\right) \bar{v}_{\phi}^{(0)}+2 \frac{r^{\prime}}{\alpha} \bar{v}_{t}^{(0)}=0, \\
2 \frac{z^{\prime}}{\alpha} \bar{v}_{\phi}^{(0)}=\frac{\partial \bar{p}^{(1)}}{\partial \eta}, \\
\frac{\partial \bar{v}_{n}^{(1)}}{\partial \eta}+\frac{1}{\alpha} \frac{\partial \bar{v}_{t}^{(0)}}{\partial s}+\frac{r^{\prime}}{r \alpha} \bar{v}_{t}^{(0)}=0 .
\end{gathered}
$$

With the boundary conditions (2.5), the two first equations immediately give

$$
\begin{aligned}
& \bar{v}_{\phi}^{(0)}=\frac{r(s)}{2}\left(e^{-\lambda_{-} \eta}+e^{-\lambda_{+} \eta}\right), \\
& \bar{v}_{t}^{(0)}=\frac{\mathrm{i} r(s)}{2}\left(e^{-\lambda_{-} \eta}-e^{-\lambda_{+} \eta}\right),
\end{aligned}
$$

where the functions

$$
\lambda_{ \pm}=\sqrt{-2 \mathrm{i}\left(\cos \theta_{c} \pm \frac{r^{\prime}}{\alpha}\right)}
$$

are such that $\Re e\left(\lambda_{ \pm}\right)>0$. The third equation (4.6c) can be used to get the pressure field, while (4.6d) gives

$$
\begin{aligned}
\bar{v}_{n}^{(1)}= & \frac{\mathrm{i} r(s) \eta}{2 \alpha}\left(\frac{\lambda_{+}^{\prime}}{\lambda_{+}} e^{-\lambda_{+} \eta}-\frac{\lambda_{-}^{\prime}}{\lambda_{-}} e^{-\lambda_{-} \eta}\right)+\left(\frac{\mathrm{i} r^{\prime}}{2 \alpha \lambda_{-}}-\frac{\mathrm{i} r \lambda_{-}^{\prime}}{2 \alpha \lambda_{-}^{2}}\right)\left(e^{-\lambda_{-} \eta}-1\right) \\
& -\left(\frac{\mathrm{i} r^{\prime}}{2 \alpha \lambda_{+}}-\frac{\mathrm{i} r \lambda_{+}^{\prime}}{2 \alpha \lambda_{+}^{2}}\right)\left(e^{-\lambda_{+} \eta}-1\right) .
\end{aligned}
$$

This last expression can be used to get the Ekman pumping in the bulk. We are especially interested in its expression close to $\mathbf{x}_{\mathbf{c}}$ when $s$ goes to $s_{c}$. Here we consider the situation illustrated on figure $7(\mathrm{~b})$, which corresponds to $L_{1}$. For this case, $s$ is always smaller than $s_{c}$. Close to $s_{c}, \lambda_{+}$remains finite but $\lambda_{-}$goes to zero as

$$
\lambda_{-} \sim(1+\mathrm{i}) \sqrt{-\kappa_{c} \alpha_{c} \sin \theta_{c}\left(s_{c}-s\right)},
$$

where $\kappa_{c}$ is the local curvature at $\mathbf{x}_{\mathbf{c}}$. The curvature $\kappa$ is defined by

$$
\kappa=\frac{z^{\prime \prime} r^{\prime}-r^{\prime \prime} z^{\prime}}{\alpha^{3}}
$$

Here, it is negative in the neighborhood of $\mathbf{x}_{\mathbf{c}}$.

If we perform the limits $\eta \rightarrow \infty$ (first) and $s \rightarrow s_{c}$ (second), we get a normal velocity which behaves as

$$
v_{n} \sim \frac{(1+\mathrm{i})}{8 \alpha_{c}^{3 / 2} \sqrt{-\kappa_{c} \sin \theta_{c}}} \frac{\sqrt{E}}{\left(s_{c}-s\right)^{3 / 2}} .
$$

This limit is valid as long as $\eta \sqrt{s_{c}-s} \rightarrow \infty$ when $s \rightarrow s_{c}$.

\subsubsection{Similarity solution near $\mathbf{x}_{\mathbf{c}}$}

The similarity solutions introduced in $\S 2.2$ are singular as we get close to $\mathbf{x}_{\mathbf{c}}$. For a fixed $\eta_{\perp}$, this amounts to consider the limit $x_{\|} \rightarrow 0$. As we are interested in the solution close to the boundary for $L_{1}$, we consider a negative $\eta_{\perp}$ [see figure $7(\mathrm{~b})$ ] such that $\zeta$ defined by (2.11) goes to $-\infty$ as $x_{\|} \rightarrow 0$. In this limit, we obtain

$$
\begin{aligned}
& v_{\|} \sim C_{0}\left(-\eta_{\perp}\right)^{-m} e^{-\mathrm{i} m \pi}, \\
& v_{\perp} \sim-C_{0} E^{1 / 3} \frac{\cos \theta_{c}}{2(m-1)}\left(-\eta_{\perp}\right)^{-m+1} e^{-\mathrm{i} m \pi} .
\end{aligned}
$$


These estimates apply as long as $-\eta_{\perp}\left(x_{\|}\right)^{-1 / 3} \gg 1$.

\subsubsection{Matching}

In this section, we show that the similarity solution and the boundary layer solution are compatible for particular values of $C_{0}$ and $m$. The matching is performed in the region where both expressions (4.12) and (4.13) apply. To determine this region, it is useful to express the variables $x_{\|}$and $\eta_{\perp}$ in terms of the boundary layer variables $s$ and $\eta$ :

$$
\begin{aligned}
& x_{\|}=-\cos \theta_{c}\left(r(s)-1-\sqrt{E} \eta \frac{z^{\prime}}{\alpha}\right)+\sin \theta_{c}\left(z(s)+\sqrt{E} \eta \frac{r^{\prime}}{\alpha}\right) \\
& \eta_{\perp}=\frac{\sin \theta_{c}}{E^{1 / 3}}\left(r(s)-1-\sqrt{E} \eta \frac{z^{\prime}}{\alpha}\right)+\frac{\cos \theta_{c}}{E^{1 / 3}}\left(z(s)+\sqrt{E} \eta \frac{r^{\prime}}{\alpha}\right)
\end{aligned}
$$

Close to $\mathbf{x}_{\mathbf{c}}$, these expressions become

$$
\begin{aligned}
& x_{\|} \sim \alpha_{c}\left(s_{c}-s\right)+\sqrt{E} \kappa_{c} \alpha_{c} \eta\left(s_{c}-s\right), \\
& \eta_{\perp} \sim E^{1 / 6} \eta+E^{-1 / 3} \kappa_{c} \alpha_{c}^{2} \frac{\left(s_{c}-s\right)^{2}}{2},
\end{aligned}
$$

where we recall that $\kappa_{c}$ is the curvature at $\mathbf{x}_{\mathbf{c}}$

If $\left|s-s_{c}\right|=O\left(E^{1 / 6}\right)$ and $E^{-1 / 12} \ll \eta \ll E^{-1 / 6}$, we do have $\sqrt{\left|s-s_{c}\right|} \eta \gg 1$ as required by (4.12). Moreover, we then get

$$
\begin{aligned}
& x_{\|} \sim \alpha_{c}\left(s_{c}-s\right), \\
& \eta_{\perp} \sim E^{-1 / 3} \kappa_{c} \alpha_{c}^{2} \frac{\left(s-s_{c}\right)^{2}}{2},
\end{aligned}
$$

which implies that $-\eta_{\perp} x_{\|}^{-1 / 3} \propto E^{-1 / 3}\left(s_{c}-s\right)^{5 / 3}=O\left(E^{-1 / 18}\right) \gg 1$ as required for the validity of (4.13). This region is materialized in red in the sketch shown in figure $7(\mathrm{~b})$. In this figure, we have also indicated the scalings of the different asymptotic regions: the $O\left(E^{1 / 2}\right)$ width of the boundary layer on the object far from $s_{c}$, the $O\left(E^{1 / 3}\right)$ width of the similarity solution around $L_{1}$ far from $s_{c}$, and the scalings in $E^{1 / 5}$ and $E^{2 / 5}$ of the local region near $s_{c}$ where the singularities of both the boundary layer solution and the similarity solution are smoothed (Roberts \& Stewartson, 1963; Kerswell, 1995).

In this region, we can then match the boundary layer solution with the similarity solution. The normal velocity $v_{n}$ can be deduced from $v_{\|}$and $v_{\perp}$ using

$$
v_{n}=\frac{1}{\alpha}\left(\left(z^{\prime} \cos \theta_{c}+r^{\prime} \sin \theta_{c}\right) v_{\|}+\left(-z^{\prime} \sin \theta_{c}+r^{\prime} \cos \theta_{c}\right) v_{\perp}\right),
$$

which becomes close to $s_{c}$

$$
v_{n} \sim v_{\perp}-\kappa_{c} \alpha_{c}\left(s_{c}-s\right) v_{\|} .
$$

If we take into account $(4.16 \mathrm{a}, \mathrm{b})$, the normal velocity obtained from the similarity solution $(4.13 \mathrm{a}-\mathrm{b})$ is therefore

$$
v_{n} \sim E^{m / 3}\left(-\kappa_{c}\right)^{-m+1} 2^{m} \alpha_{c}^{-2 m+1}\left(s_{c}-s\right)^{-2 m+1} C_{0} e^{-\mathrm{i} m \pi} .
$$

This expression is compatible with (4.12) if and only if

$$
\begin{aligned}
& m=5 / 4, \\
& C_{0}=\frac{E^{1 / 12}}{8 \sqrt{2 \sin \theta_{c}}\left(-2 \kappa_{c}\right)^{1 / 4}} e^{-\mathrm{i} \pi / 2} .
\end{aligned}
$$




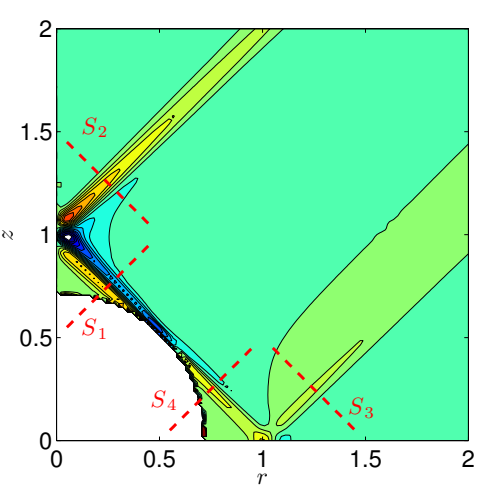

(a)

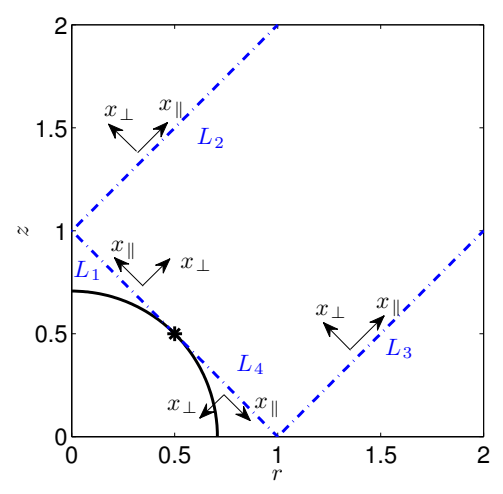

(b)

Figure 8. (a) Contour of the azimuthal velocity $v_{\phi}$ in the $(r, z)$ plane of a librating sphere obtained from the numerical simulation at time $t=508.9 \pi / \omega$ for $E_{s}=10^{-5}, \varepsilon=10^{-4}, \omega=\sqrt{2}$. The sections $S_{j}, j=1,2,3,4$ where the profiles are analysed in figure 9 are also indicated. (b) Definition of the local coordinate system on the characteristic lines $L_{j}, j=1,2,3,4$. The star indicates the source point of the similarity solutions.

These expressions for $m$ and $C_{0}$ apply for the similarity solution along the line $L_{1}$. A similar analysis can be carried out for the similarity solution along $L_{4}$ by performing the matching on the other side of $\mathbf{x}_{\mathbf{c}}$. It gives the same value of $m$ and an amplitude $C_{0}$ just different by a phase factor:

$$
C_{0}=\frac{E^{1 / 12}}{8 \sqrt{2 \sin \theta_{c}}\left(-2 \kappa_{c}\right)^{1 / 4}} e^{-\mathrm{i} \pi / 4} .
$$

These values of $m$ and $C_{0}$ constitute the main result of the present paper. In particular, it is worth mentioning that the value of $m$ is larger for a smooth surface (spheroid) than for an angular one (disk). This means that we have the following counter-intuitive result: the similarity solution describing the internal shear layer is associated with a stronger singularity at $\mathbf{x}_{\mathbf{c}}$ for a smooth surface than for an angular one. As a consequence, the amplitude of the velocity field is larger for the spheroid with a scaling in $E^{1 / 12}$ to be compared with the $E^{1 / 6}$ scaling for the disk. Expressions (4.20b) and (4.21) provide the dependency with respect to the libration frequency through the term $2 \sin \theta_{c}=\sqrt{4-\omega^{2}}$. The amplitude of the internal shear layer increases when $\theta_{c}$ decreases, that is when $\omega$ gets close to 2 ( or $\omega^{*}$ close to $2 \Omega^{*}$ ). We can also note the dependency with respect to the local curvature $\kappa_{c}$. The amplitude decreases as $\left|\kappa_{c}\right|$ increases. The more peaky the surface at $\mathbf{x}_{\mathbf{c}}$ is, the smaller the amplitude of the internal shear layer is. This is consistent with the smaller amplitude scaling obtained for the singular case of the disk.

\subsection{Comparison with numerical results}

In this section, the formulas $(4.20 \mathrm{a}, \mathrm{b})$ are tested against direct numerical simulations. Different spheroids as well as different frequencies are considered.

The numerical simulations were first performed for a sphere of radius $1 / \sqrt{2}$ with a frequency $\omega=\sqrt{2}$ such that the singular rays start from the point $\mathbf{x}_{\mathbf{c}}=(1 / 2,1 / 2)$ on the surface and cross the meridional plane and the rotation axis at $(1,0)$ and $(0,1)$ respectively, as illustrated in figure $8(\mathrm{a})$. This normalisation does not correspond to that chosen for the similarity solution. The Ekman number of the simulation $E_{s}$ is therefore 4 times smaller than the Ekman number defined in (2.2). The internal shear layers are 

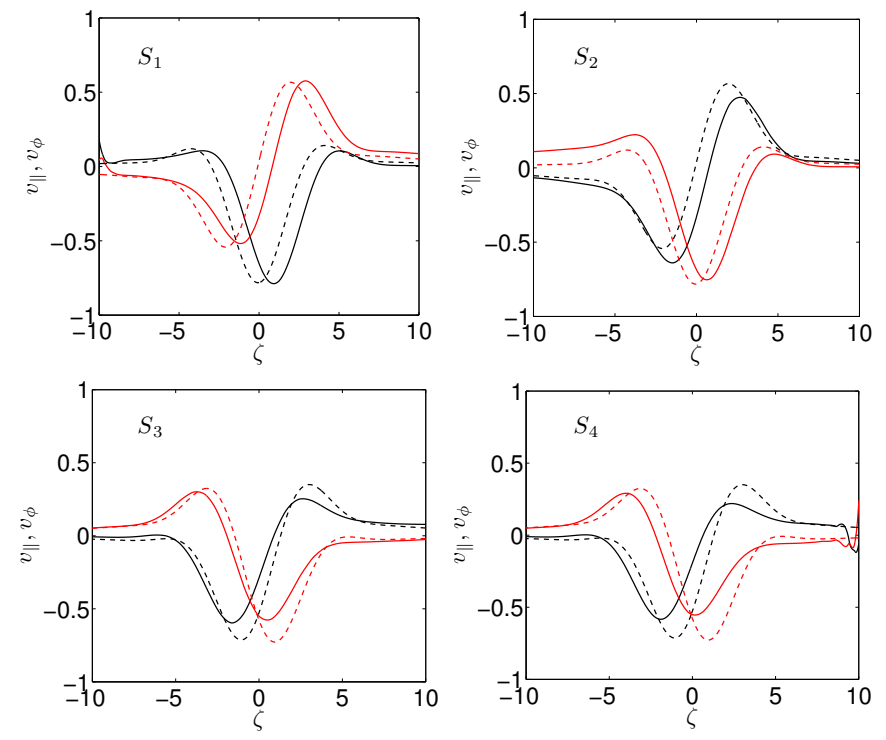

Figure 9. Profiles of $v_{\phi}$ (red curves) and $v_{\|}$(black curves) across the internal shear layer in the sections $S_{1}$ (top left), $S_{2}$ (top right), $S_{3}$ (bottom left) and $S_{4}$ (bottom right) as indicated in figure 8 , at time $t=508.9 \pi / \omega$ for $E_{s}=2.510^{-6}$ (i.e. $E=10^{-5}$ ), $\varepsilon=10^{-4}, \omega=\sqrt{2}$. Solid lines are numerical results. Dashed lines are the similarity solutions. All the solutions have been renormalized by the factor $A=\varepsilon\left|C_{0}\right|\left(2 \sin \theta_{c} / x_{\|}\right)^{5 / 4} / \sqrt{r}$ with $C_{0}$ given by (4.20b) or (4.21).

emitted on either side of $\mathbf{x}_{\mathbf{c}}$ along the lines $L_{1}$ and $L_{4}$ indicated in figure $8(\mathrm{~b})$. The internal shear layer on $L_{1}$ is reflected on the axis and continues along $L_{2}$. The internal shear layer on $L_{3}$ corresponds to that emitted from the symmetric point $(1 / 2,-1 / 2)$ on the sphere. The definition of the coordinate systems is given in figure $8(\mathrm{~b})$, in agreement with the definition used in figure 1.

In figure 9, we compare the numerical solution to the similarity solution in the sections indicated in figure 8(a) at a particular instant. Both the azimuthal velocity and the parallel velocity are plotted as a function of the similarity variable. As for the disk, the normalisation has been chosen such that the theoretical predications for $v_{\phi}$ and $v_{\|}$are just given by

$$
V_{\|}^{S S}=\Re e\left(h_{5 / 4}(\zeta) e^{-\mathrm{i} \varphi_{\|}}\right), \quad V_{\phi}^{S S}=\Im m\left(h_{5 / 4}(\zeta) e^{-\mathrm{i} \varphi_{\phi}}\right)
$$

where

$$
\begin{aligned}
& \varphi_{\|}=\varphi_{\phi}=\omega t_{o}+\pi / 2 \text { for } S_{1} \\
& \varphi_{\|}=\varphi_{\phi}+\pi=\omega t_{o} \text { for } S_{2}, \\
& \varphi_{\|}=\varphi_{\phi}+\pi=\omega t_{o}+\pi / 4 \text { for } S_{3} \text { and } S_{4} .
\end{aligned}
$$

As for the disk, we have also looked at the solution along the characteristic lines $L_{j}$. For the spheroid, we expect $\left|x_{\|}\right|^{5 / 12} \sqrt{r}|\mathbf{v}|$ to remain constant and uniform and given by

$$
\left|x_{\|}\right|^{5 / 12} \sqrt{r}|\mathbf{v}| \sim \varepsilon\left|h_{5 / 4}(0)\right|\left|C_{0}\right|\left(2 \sin \theta_{c}\right)^{5 / 12} \approx 0.0776 \frac{\varepsilon E^{1 / 12}}{\left(\sin \theta_{c}\right)^{1 / 12}\left|\kappa_{c}\right|^{1 / 4}} .
$$

This is verified in figure 10 where we have plotted $\left|x_{\|}\right|^{5 / 12} \sqrt{r}|\mathbf{v}|$ versus $x_{\|}$along the lines $L_{1}$ and $L_{2}$ (top) and $L_{4}$ and $L_{3}$ (bottom) for 25 equidistant times within a period. The theoretical prediction (4.24) is indicated as a red dashed line. The agreement is good for 

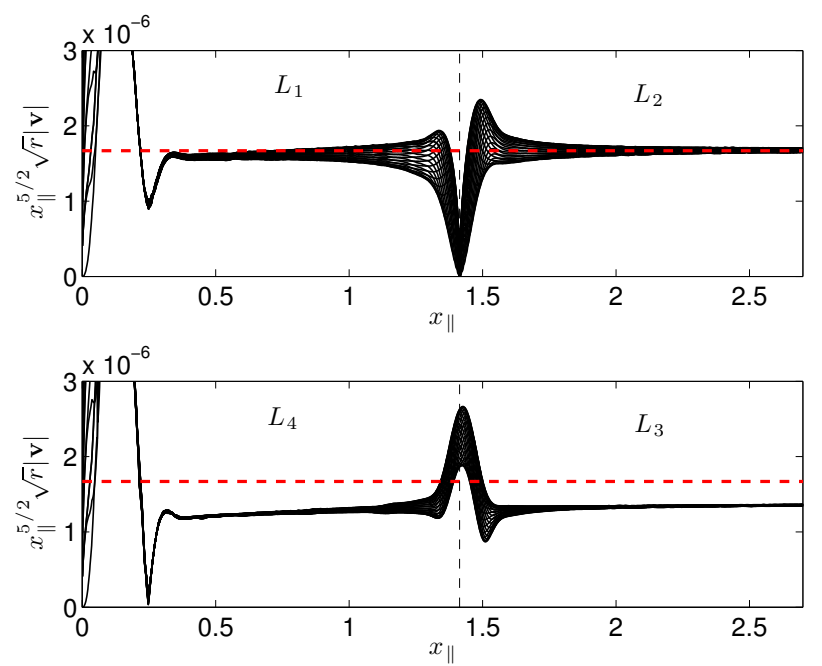

FIgURE 10. Norm of the velocity vector along the characteristic lines $L_{j}$ normalized by $x_{\|}^{-5 / 2} r^{-1 / 2}$ for a librating sphere with $E_{s}=2.510^{-6}$ (i.e. $E=10^{-5}$ ), $\varepsilon=10^{-4}$, $\omega=\sqrt{2}, \theta_{c}=\pi / 4$. The solid black lines are the numerical results obtained for time $\omega t /(2 \pi)=250: 1 / 25: 251$. The dashed red line is the theoretical prediction (4.24).

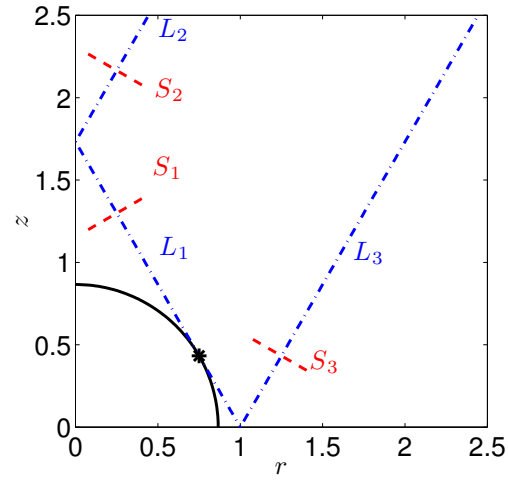

(a)

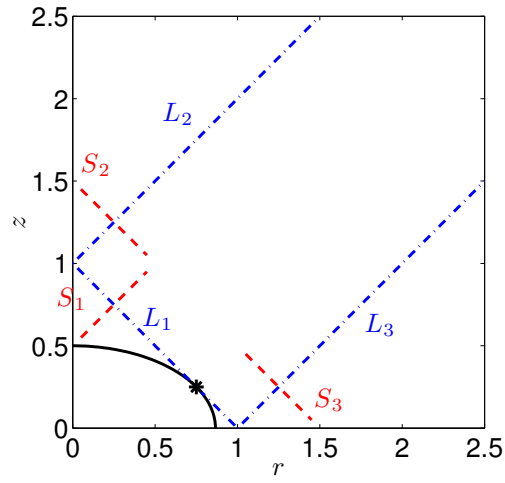

(b)

FiguRE 11. Definition of the characteristic lines and sections for a librating spheroid. (a) Sphere of radius $\sqrt{3} / 2$ for $\omega=1$. (b) Oblate spheroid of eccentricity $\sqrt{2 / 3}$ for $\omega=\sqrt{2}$.

the upper lines $L_{1}$ and $L_{2}$. However, as for the disk, there is a weak discrepancy between the theory and the numerics for the constant on the lower lines $L_{4}$ and $L_{3}$. Close to $x_{\|} \approx \sqrt{2}$, the departures are associated with the impact of $L_{1}$ and $L_{2}$ on the axis, and with the crossing of $L_{4}$ and $L_{3}$.

Other geometries have been tested numerically. We present here two other configurations, a sphere with a different frequency and an oblate spheroid (see figure 11). For both cases, we have performed the same tests. In figure 12, we provide plots of $v_{\phi}$ and $v_{\|}$in different sections indicated in figure 11 using the same normalisation as above such that formulas (4.22) still apply. In figure 13, we have plotted as in figure 10, the quantity $\left|x_{\|}\right|^{5 / 12} \sqrt{r}|\mathbf{v}|$ versus $x_{\|}$, which is still expected to be provided by (4.24). As previously, 

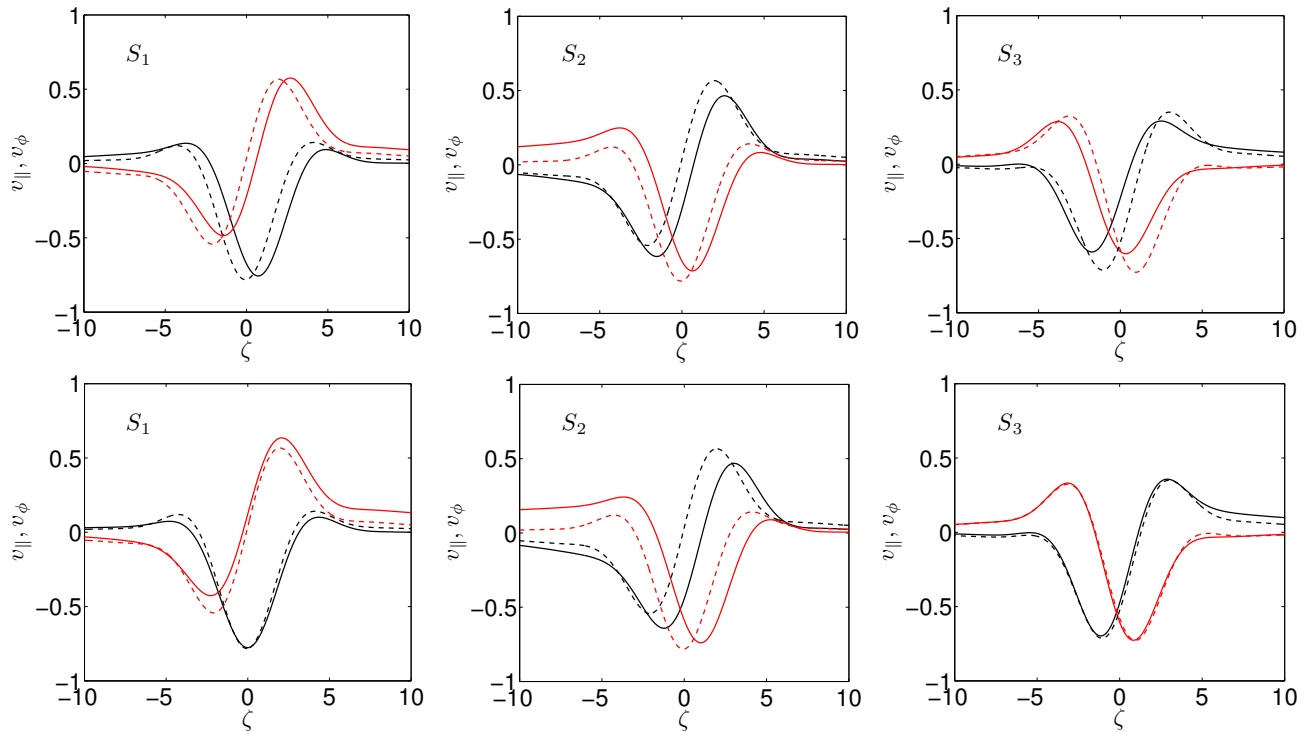

FiguRE 12. Same as figure 9 across the sections defined in figure 11. Top: sphere with $\omega=1$; bottom: oblate spheroid of eccentricity $\sqrt{2 / 3}$ with $\omega=\sqrt{2}$. For both cases, $\varepsilon=10^{-4}, E_{s}=10^{-5}$ (i.e. $E=1.7810^{-5}$ ) and $t=508.9 \pi / \omega$.
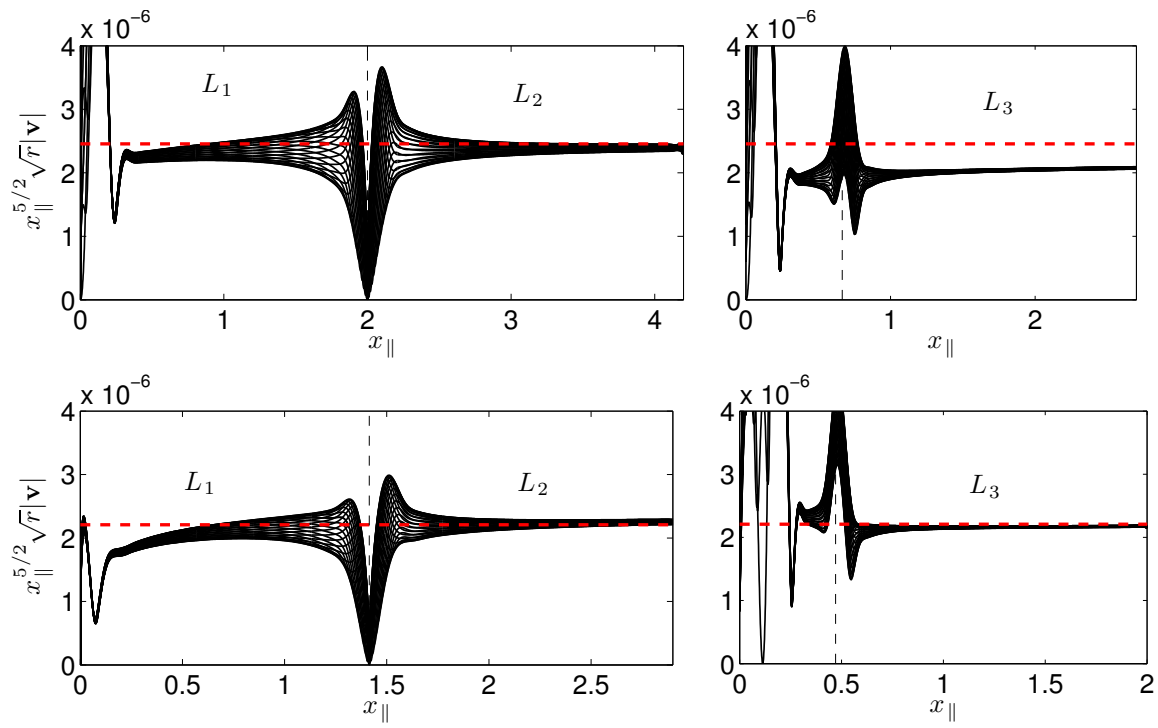

FIGURE 13. Same as figure 10 for the configurations defined in figure 11. Top: sphere with $\omega=1$; bottom: oblate spheroid of eccentricity $\sqrt{2 / 3}$ with $\omega=\sqrt{2}$. For both cases, $\varepsilon=10^{-4}$ and $E_{s}=10^{-5}$ (i.e. $E=1.7810^{-5}$ ).

we do observe a good agreement between theoretical predictions and numerical results.

\section{Discussion}

We have shown that the internal shear layers created by the libration of a convex object can be described by the similarity solutions introduced by Moore \& Saffman (1969). We 


\begin{tabular}{c|c|c|c|c}
$\left|\Omega^{*}(\mathrm{rad} / \mathrm{s})\right|$ & $r^{*}(\mathrm{~km})$ & $\omega$ & $\varepsilon$ & $E$ \\
\hline Mercury $\left|1.2 \times 10^{-6}\right| \begin{array}{c}1000 \\
(\text { from } 0 \text { to } 1700)\end{array}$ & $|2 / 3| 1.3 \times 10^{-4} \mid 8 \times 10^{-13}$ \\
\hline Enceladus $\left|5.2 \times 10^{-5}\right|$ & 200 & 1 & $\left|2.1 \times 10^{-3}\right| 5 \times 10^{-13}$
\end{tabular}

TABle 1. Physical parameters for the librating motion of the liquid core of Mercury (Margot et al., 2007; Noir et al., 2009) and the subsurface ocean of Enceladus (Thomas et al., 2016). In both cases, we consider a typical viscosity $\nu=10^{-6} \mathrm{~m}^{2} / \mathrm{s}$, which is supposedly representative of both water/salty water in oceans and liquid iron in planetary cores (see e.g. de Wijs et al., 1998).

have provided the amplitude and the parameter controlling the form of the solution for a disk and for any smooth object. For a smooth object, we have in particular shown that the amplitude is proportional to the local curvature radius at the power $1 / 4$ at the critical latitude where the internal shear layers are emitted. Interestingly, we have also obtained that this amplitude is larger for a smooth object (with a scaling in $E^{1 / 12}$ ) than for a disk (with a scaling in $E^{1 / 6}$ ). The consequence of this larger amplitude in the internal shear layers is an even larger amplitude in $E^{-1 / 12}$ at the point of focus on the axis (see appendix). At this particular point on the axis, the solution therefore grows and diverges as $E \rightarrow 0$, clearly demonstrating the singular character of the solution in this limit.

The scaling in $E^{1 / 12}$ for the velocity amplitude in the internal shear layers is larger than that reported in the literature (e.g. Kerswell, 1995; Calkins et al., 2010). As a consequence, the zonal flow created by the self-interaction of the harmonic solution with itself is also expected to be larger. Following the analysis performed in Le Dizès (2015), we can expect a zonal flow in $\varepsilon^{2} E^{1 / 6}$ in the internal shear layers and in $\varepsilon^{2} E^{-1 / 2}$ at the focus point on the axis. This last scaling implies that the nonlinear corrections become large as soon as $\varepsilon$ reaches $E^{1 / 4}$, that is well before the linear solution has reached an amplitude of order 1 .

For illustrating the geophysical relevance of our results, let us consider two examples: the liquid core of Mercury and the subsurface ocean of Enceladus, whose existences have been demonstrated by studies of their surfacic librating motions (Margot et al., 2007; Thomas et al., 2016). Relevant physical parameters are listed in table 1. Using our scaling law (4.24), typical velocities within internal shear layers are $1.3 \times 10^{-6}$ and $1.6 \times 10^{-4} \mathrm{~m} / \mathrm{s}$, while estimates for the zonal flow at the focus point give $2.0 \times 10^{-2}$ and $6.3 \mathrm{~m} / \mathrm{s}$. For comparison, typical velocities in the Earth's core related to the convective dynamo are $10^{-4} \mathrm{~m} / \mathrm{s}$ (Gubbins \& Roberts, 1987) and typical tidal speeds in the Earth's oceans are $3 \times 10^{-2} \mathrm{~m} / \mathrm{s}$ (St Laurent \& Garrett, 2002). The influence of the fluid motions described here on planetary dynamics, for instance through magnetic field generation and energy dissipation, should thus be taken into account.

We have only considered internal shear layers created by libration of an axisymmetric object, hence created by viscous coupling. This forcing is weak as it is actually associated with an oscillating Ekman pumping of order $E^{1 / 2}$. A larger forcing is obtained when it is generated by pressure forces. For example, if the object was oscillated vertically or horizontally, or when the librating object is non-axisymmetric, as tidally deformed planetary bodies, a much larger amplitude would have been obtained. This situation has been analysed in several works in the context of stratified fluids (e.g. Mowbray \& 
Rarity, 1967). In these cases, internal shear layers have also been observed and described by the same family of similarity solutions in the far field (e.g. Thomas \& Stevenson, 1972; Hurley \& Keady, 1997; Flynn et al., 2003; Voisin, 2003). It would be interesting to determine whether the present approach can be also used to characterise the near field.

It is finally worth emphasizing that we have considered an open domain only. In a closed geometry such as a spherical shell, the internal shear layers are reflected on the boundaries. This implies that they can interact with themselves after a finite number of reflections modifying their internal structure. Such a modification was already quantified a long time ago for the stationary flow generated between two differentially rotating spheres by Stewartson (1966), who showed that the structure of the internal shear layer becomes more complex with nested regions of widths $E^{1 / 3}, E^{2 / 7}$ and $E^{1 / 4}$. We can imagine that similar complications would occur for the librating case if the internal shear layer would form a closed circuit.

\section{Acknowledgments}

This work received support from the French Agence Nationale de la Recherche under the A*MIDEX grant ANR-11-IDEX-0001-02, the LABEX MEC project ANR-11-LABX0092 and the ANR LIPSTIC project ANR-13-JS05-0004-01.

\section{Appendix A. Local asymptotic solution for the internal shear layer close to the axis}

The similarity solution diverges as $r$ goes to zero. A new expression has to be obtained close to the axis. For this purpose, we can use the general expression in terms of the Hankel transform

$$
\begin{aligned}
& v_{r}=\int_{0}^{\infty} U(k) J_{1}(k r) e^{\mathrm{i} \mu_{1} z} d k, \\
& v_{\phi}=\int_{0}^{\infty} V(k) J_{1}(k r) e^{\mathrm{i} \mu_{1} z} d k, \\
& v_{z}=\int_{0}^{\infty} W(k) J_{0}(k r) e^{\mathrm{i} \mu_{1} z} d k, \\
& p=\int_{0}^{\infty} P(k) J_{0}(k r) e^{\mathrm{i} \mu_{1} z} d k,
\end{aligned}
$$

where $\mu_{1}$ is the "non-viscous" wavenumber, which expands, when $E \rightarrow 0$, as

$$
\mu_{1} \sim k \frac{\cos \theta_{c}}{\sin \theta_{c}}\left(1+\mathrm{i} E \frac{k^{2}}{2 \cos \theta_{c} \sin ^{4} \theta_{c}}\right) .
$$

At leading order, the amplitudes $U, V, W$ and $P$ are related with each other by the relations (see Le Dizès, 2015)

$$
\begin{array}{r}
W=\mathrm{i} \frac{\sin \theta_{c}}{\cos \theta_{c}} U, \\
U=\mathrm{i} \cos \theta_{c} V, \\
k P=-2 V-2 \mathrm{i} \cos \theta_{c} U=-2 \sin \theta_{c} V .
\end{array}
$$

Introducing the local variables

$$
\tilde{r}=r / E^{1 / 3}, \tilde{z}=\left(z-\sin \theta_{c}\right) / E^{1 / 3},
$$


(A 1a-d) can be written as

$$
\begin{aligned}
& v_{r}=\mathrm{i} \cos \theta_{c} \int_{0}^{\infty} \tilde{V} J_{1}(\tilde{k} \tilde{r}) e^{\mathrm{i} \tilde{k} \operatorname{cotan} \theta_{c} \tilde{z}} d \tilde{k} \\
& v_{\phi}=\int_{0}^{\infty} \tilde{V} J_{1}(\tilde{k} \tilde{r}) e^{\mathrm{i} \tilde{k} \operatorname{cotan} \theta_{c} \tilde{z}} d \tilde{k} \\
& v_{z}=-\sin \theta_{c} \int_{0}^{\infty} \tilde{V} J_{0}(\tilde{k} \tilde{r}) e^{\mathrm{i} \tilde{k} \operatorname{cotan} \theta_{c} \tilde{z}} d \tilde{k} \\
& p=-2 \sin \theta_{c} E^{1 / 3} \int_{0}^{\infty} \tilde{V} \frac{J_{0}(\tilde{k} \tilde{r})}{\tilde{k}} e^{\mathrm{i} \tilde{k} \operatorname{cotan} \theta_{c} \tilde{z}} d \tilde{k} .
\end{aligned}
$$

The expression of $\tilde{V}$ is obtained by matching these expressions with the similarity solution on $L_{1}$. When both $\tilde{z}$ and $\tilde{r}$ go to infinity, (A 5b) for $v_{\phi}$ can be written as

$$
v_{\phi} \sim \sqrt{\frac{1}{2 \pi \tilde{r}}} \int_{0}^{\infty} \frac{\tilde{V}}{\sqrt{\tilde{k}}}\left(e^{\mathrm{i} \tilde{k}\left(\tilde{r}+\operatorname{cotan} \theta_{c} \tilde{z}\right)-3 \mathrm{i} \pi / 4}+e^{\mathrm{i} \tilde{k}\left(-\tilde{r}+\operatorname{cotan} \theta_{c} \tilde{z}\right)+3 \mathrm{i} \pi / 4}\right) d \tilde{k},
$$

using the expansion of the Bessel function $J_{1}$ at infinity. If we now assume that

$$
\tilde{V}=E^{-1 / 6} C_{0} \frac{\sqrt{2 \pi} \tilde{k}^{m-1 / 2}}{(m-1) ! \sin ^{m} \theta_{c}} e^{-\tilde{k}^{3} /\left(2 \cos \theta_{c} \sin ^{4} \theta_{c}\right)} e^{5 \mathrm{i} \pi / 4-\mathrm{i} m \pi / 2},
$$

this expression becomes

$$
v_{\phi} \sim \frac{1}{\sqrt{r}}\left(\mathrm{i} C_{0} H_{m}\left(1 / \cos \theta_{c}, \zeta^{(1)}\right)+C_{0} H_{m}\left(1 / \cos \theta_{c}, \zeta^{(2)}\right)\right)
$$

where $\zeta^{(1)}$ and $\zeta^{(2)}$ are the similarity variables close to the axis along $L_{1}$ and $L_{2}$ respectively:

$$
\begin{aligned}
& \zeta^{(1)}=\left(2 \sin \theta_{c} \cos \theta_{c}\right)^{1 / 3}\left(\sin \theta_{c} \tilde{r}+\cos \theta_{c} \tilde{z}\right), \\
& \zeta^{(2)}=\left(2 \sin \theta_{c} \cos \theta_{c}\right)^{1 / 3}\left(-\sin \theta_{c} \tilde{r}+\cos \theta_{c} \tilde{z}\right) .
\end{aligned}
$$

The first term in (A 8) is exactly the expression of the azimuthal velocity component of the similarity solution along $L_{1}$ close to $x_{\|}=1 / \cos \theta_{c}$ [see expressions (2.8) and (2.15)]. The second term corresponds to the similarity solution along $L_{2}$. It shows that the azimuthal velocity component of the similarity solution keeps the same amplitude but with a phase shift of $-\pi / 2$. Along $L_{2}$, the expression $(2.10)$ and $(2.15)$ for $v_{\|}^{(0)}$ and $v_{\phi}^{(0)}$ have thus to be replaced by

$$
v_{\|}^{(0)}=\mathrm{i} C_{0} H_{m}\left(x_{\|}, \zeta\right), \quad v_{\phi}^{(0)}=C_{0} H_{m}\left(x_{\|}, \zeta\right),
$$

where $x_{\|}$starts at the value $1 / \cos \theta_{c}$ from the point $\left(0, \tan \theta_{c}\right)$ on the $z$-axis.

In the $O\left(E^{1 / 3}\right)$ neighborhood around the focus point, the solution is given by (A $\left.5 \mathrm{a}-\mathrm{d}\right)$ with $\tilde{V}$ and $C_{0}$ provided by (A 7$)$ and $(4.20 \mathrm{~b})$, respectively. In particular, this implies that the three velocity components become large of order $E^{-1 / 12}$ close to the focus point.

\section{References}

Aldridge, K. D. \& Toomre, A. 1969 Axisymmetric inertial oscillations of a fluid in a rotating spherical container. J. Fluid Mech. 37, 307-323.

Calkins, M. A., Noir, J., Eldredge, J. D. \& Aurnou, J. M. 2010 Axisymmetric simulations of libration-driven fluid dynamics in a spherical shell geometry. Phys. Fluids 22, 086602. 
Cébron, D., Le Bars, M., Moutou, C. \& Le Gal, P. 2012 Elliptical instability in terrestrial planets and moons. Astronomy \& Astrophysics 539, A78.

Flynn, M. R., Onu, K. \& Sutherland, B. R. 2003 Internal wave excitation by a vertically oscillating sphere. J. Fluid Mech. 494, 65-93.

Greenspan, H. P. 1968 The theory of rotating fluids. Cambridge University Press.

Gubbins, D. \& Roberts, P. H. 1987 Magnetohydrodynamics of the earth's core. Geomagnetism 2, 1-183.

Hurley, D. G. \& KeAdy, G. 1997 The generation of internal waves by vibrating elliptic cylinders. Part 2. Approximate viscous solution. J. Fluid Mech. 351, 119-138.

KeRswelL, R. 1995 On the internal shear layers spawned by the critical regions in oscillatory Ekman boundary layers. J. Fluid Mech. 298, 311-325.

KIDA, S. 2011 Steady flow in a rapidly rotating sphere with weak precession. J. Fluid Mech. 680, 150-193.

Klein, M., Seelig, T., Kurgansky, M., Ghasemi V., A. et al. 2014 Inertial wave excitation and focusing in a liquid bounded by a frustrum and a cylinder. J. Fluid Mech. 751, 255-297.

Koch, S., Harlander, U., Egbers, C. \& Hollerbach, R. 2013 Inertial waves in a spherical shell induced by librations of the inner sphere: experimental and numerical results. Fluid Dyn. Research 45 (3), 035504.

Le Bars, M., CÉBron, D. \& Le Gal, P. 2015 Flows driven by libration, precession, and tides. Annu. Rev. Fluid Mech. 47, 163-193.

LE Diż̀s, S. 2015 Wave field and zonal flow of a librating disk. J. Fluid Mech. 782, $178-208$.

Machiconne, N., Cortet, P.-P., Voisin, B. \& Moisy, F. 2015 Influence of the multipole order of the source on the decay of an inertial wave beam in a rotating fluid. Phys. Fluids 27, 066602.

Marcotte, F., Dormy, E. \& Soward, A. 2016 On the equatorial Ekman layer. J. Fluid Mech. 803, 395-435.

Margot, J.-L., Peale, S. J., Jurgens, R. F., Slade, M. A. \& Holin, I. V. 2007 Large longitude libration of mercury reveals a molten core. Science $\mathbf{3 1 6}$ (5825), 710-714.

MCEwAn, A. D. 1970 Inertial oscillations in a rotating fluid cylinder. J. Fluid Mech. 40, 603-640.

Moore, D. W. \& Saffman, P. G. 1969 The structure of free vertical shear layers in a rotating fluid and the motion produced by a slowly rising body. Phil. Trans. R. Soc. A 264, 597-634.

Mowbray, D. E. \& Rarity, B. S. H. 1967 A theoretical and experimental investigation of the phase configuration of internal waves of small amplitude in a density stratified liquid. J. Fluid Mech. 28, 1-16.

Noir, J., Hemmerlin, F., Wicht, J., BacA, S. M. \& Aurnou, J. M. 2009 An experimental and numerical study of librationally driven flow in planetary cores and subsurface oceans. Phys. Earth Planet. Inter. 173 (1), 141-152.

OgILVIE, G. I. 2005 Wave attractors and the asymptotic dissipation rate of tidal disturbances. J. Fluid Mech. 543, 19-44.

PEAT, K. S. 1978 Internal and inertial waves in a viscous rotating stratified fluid. Appl. Sci. Res 33, 481-499.

Proudman, I. 1956 The almost-rigid rotation of viscous fluid between concentric spheres. J. Fluid Mech. 1, 505-516.

Rieutord, M., Georgeot, B. \& Valdettaro, L. 2001 Inertial waves in a rotating spherical shell: attractors and asymptotic spectrum. J. Fluid Mech. 435, 103-144. 
Rieutord, M. \& Valdettaro, L. 1997 Inertial waves in a rotating spherical shell. J. Fluid Mech. 341, 77-99.

Roberts, P. H. \& Stewartson, K. 1963 On the stability of a MacLaurin spheroid of small viscosity. Astrophys. J. 137, 777-790.

St Laurent, L. \& Garrett, C. 2002 The role of internal tides in mixing the deep ocean. J. Phys. Oceanography 32 (10), 2882-2899.

Stevenson, T. N., Bearon, J. N. \& Thomas, N. H. 1974 An internal wave in a viscous heat-conducting isothermal atmosphere. J. Fluid Mech. 65, 315-323.

Stewartson, K. 1957 On almost rigid rotations. J. Fluid Mech. 3, 17-26.

Stewartson, K. 1966 On almost rigid rotations. Part 2. J. Fluid Mech. 26, 131-144.

Thomas, N. H. \& Stevenson, T. N. 1972 A similarity solution for viscous internal waves. J. Fluid Mech. 54, 495-506.

Thomas, P. C., Tajeddine, R., Tiscareno, M. S., Burns, J. A., Joseph, J., Loredo, T. J., Helfenstein, P. \& Porco, C. 2016 Enceladus's measured physical libration requires a global subsurface ocean. Icarus 264, 37-47.

Tilgner, A. 2000 Oscillatory shear layers in source driven flows in an unbounded rotating fluid. Phys. Fluids 12, 1101-11.

Voisin, B. 2003 Limit states of internal wave beams. J. Fluid Mech. 496, 243-293.

VAN DE VoOREn, A. I. 1992 The Stewartson layer of a rotating disk of finite radius. $J$. Eng. Math. 26, 131-152.

Walton, I. C. 1975 On waves in a thin rotating spherical shell of slightly viscous fluid. Mathematika 22, 46-59.

De Wijs, G. A., Kresse, G., Vocadlo, L., Dobson, D. et al. 1998 The viscosity of liquid iron at the physical conditions of the earth's core. Nature 392 (6678), 805.

Zhang, K., Chan, K. H., Liao, X. \& Aurnou, J. M. 2013 The non-resonant response of fluid in a rapidly rotating sphere undergoing longitudinal libration. J. Fluid Mech. $\mathbf{7 2 0}, 212$. 